\title{
Manual of Neonatal Respiratory Care - Third Edition
}

\author{
Steven M. Donn, Sunil K. Sinha. Springer, New York, 2012, 787 pages, \\ ISBN 978-1-4614-2154-2
}

\author{
Eric D. Greenwood, MD
}

Received: 28 November 2012/Accepted: 5 December 2012/Published online: 3 January 2013

(c) Canadian Anesthesiologists' Society 2012

The Manual of Neonatal Respiratory Care offers a comprehensive review of the respiratory physiology, ventilation methods, and respiratory diseases of the newborn. This third edition text is a thorough update of the second edition published in 2006. The focus of the text is on the neonate, but many of the principles reviewed apply to all patients from newborn to adult. This book will serve as an excellent basic science review for the practicing anesthesiologist who cares for pediatric patients as well as a good source of information for physicians in training. The content presented is applicable to anesthesiology practice; however, our specialty is a secondary audience as no content is written specifically on the unique challenges faced in the operating room.

Contributing authors are from the United States, Canada, and the United Kingdom, with many from the University of Michigan. Most contributors are engaged in the pediatric subspecialties, including neonatology, respirology, and general surgery, which presents a moderately diverse perspective.

The text is divided into 15 parts with 85 chapters. Fortunately, the well-organized table of contents simplifies finding topics of interest in this large text. The chapters are grouped logically and appropriately; they are well written and include appropriate figures and tables. Where duplication of content occurs due to contributions from different authors, the repetition is constructive.

E. D. Greenwood, MD ( $\varangle)$

The Hospital for Sick Children, University of Toronto, Toronto,

ON, Canada

e-mail: eric.greenwood@ sickkids.ca

E. D. Greenwood, MD

Brampton Civic Hospital, William Osler Health System,

Brampton, ON, Canada
Part I of the text begins with a review of embryology and is followed by an extensive review of respiratory physiology, which is well presented and applicable to all patients from newborn to adult. This first section is one of the sections from which anesthesiologists will benefit most. Topics in the next sections encompass procedures, monitoring, and non-invasive ventilation, material that is less pertinent to our specialty. Part II is devoted to ventilation, including conventional and high-frequency ventilation. This is a good review of conventional ventilation followed by excellent details on high-frequency oscillatory ventilation and jet ventilation. These chapters provide an excellent overview of high-frequency ventilation techniques and offer a good reference for practitioners who are unfamiliar with these non-conventional ventilation techniques. Further chapters are dedicated to topics such as nitric oxide therapy and extracorporeal membrane oxygenation with wellpresented overviews of these techniques. Fourteen chapters are dedicated to neonatal respiratory diseases. Relevant disorders considered in these chapters include congenital diaphragmatic hernia, respiratory distress syndrome, and bronchopulmonary dysplasia.

Further sections present topics on complications associated with mechanical ventilation, including relevant topics such as patent ductus arteriosus and retinopathy of prematurity. Adjunctive care, such as hemodynamic support, is presented in further chapters.

Additional chapters discuss topics relating to neonatal intensive care, such as nutrition, sedation, and surfactant replacement. These topics are likely outside our specialty's area of interest. In addition, an entire section of the text is dedicated to specific ventilators commonly used in the neonatal intensive care unit. The book concludes with sections on ethics, legal considerations, and research in neonatal care. 
In summary, this text, which was written predominately for the neonatologist, will provide an impressive review and reference for the anesthesiologist who cares for neonates. Details on respiratory physiology are more detailed and specific to the neonate than would be included in a text on general anesthesia. The chapters on topics such as high-frequency ventilation offer detailed explanations on non-conventional ventilation, which is frequently a mystery to many anesthesiologists. I would recommend including this text in the library of any anesthesiology department that provides anesthetics to infants.

Competing interests None declared. 